\title{
Augmented Space Recursion Method for the Study of Electronic States of Binary Alloys
}

\author{
M. Abdus Salam*a, Kabir Ahmed ${ }^{\mathrm{a}}$, B. P. Barua ${ }^{\mathrm{b}}$, and M. S. I Aziz \\ ${ }^{a}$ BCSIR Laboratories, Chittagong-4220, Bangladesh and ${ }^{b}$ Department of Physics \\ University of Chittagong, Bangladesh
}

\begin{abstract}
We have studied here the electronic structure of pure random disordered alloys formed by Ni with $\mathrm{Cu}$ and Au at different ratios by using the linearized tight-binding muffin-tin Orbital (TB-LMTO) method. We also used the recursion technique together with augmented space formalism for increasing the efficiency and the accuracy to calculate the component projected density of states. From the density of state, we can understand the Fermi energy, magnetic moment and binding energy at different alloy compositions. The band structure can be calculated from here also. These studies are helpful for experimentalists and metallurgists in designing materials and alloys with specific properties.
\end{abstract}

Key words: Electronic structure, Alloys, TB-LMTO, Density of states, Augmented space recursion

\section{Introduction}

Among the solid materials, metals are of great interest. For example iron is used in automobiles, copper in electric wiring, where as gold and silver are used in jewelry. These and other metals have played a very important role in the development of our technological world from early historical time to the present and will continue to do so in the future. The explanation of characteristic metallic properties is important to a metallurgist or an engineer who wishes to use metals for practical purposes and to a physicist who is interested in understanding the microscopic structure of materials.

The tight-binding or screened version of the linearized muffin-tin-orbitals method (TB-LMTO) has provided an excellent starting point for first principles electronic structure determination of disordered alloys. The resulting Hamiltonian is short-ranged and therefore ideally provides the extension from the traditional adhoc tight-binding ideas into a fully-self-consistent first principles theory. The basis of the method has been described in detail (Andersen et al., 1994) and relevant details necessary for disordered alloys are given in (Anderson and Japsen et al.,1984). The study of electronic structure of disordered alloys is of great scientific and technological importance. Theoretical approaches have achieved considerable success through the development of mean-field approximations, the most successful of which is the coherent potential approximation (CPA) (Pinski, et al., 1991). Other techniques include super-cell approaches, attempted generalizations of the CPA and an alternative order-N Green's function technique (Abrikosov et al., 1996). The former is based on the self-consistent determination of a uniform medium to represent the substitutional alloy. The corresponding effective Hamiltonian is lattice transitionally symmetric and its Green function is a good approximation of the configurational averaged Green function. Of the later, the super-cell method is based on the study of different selected ordered structures at various concentrations. In these calculations a large unit cell is constructed which contains different possible configurations and is repeated to generate the entire lattice. The result of such a method contains the artifact of imposed lattice symmetry, which is OK provided we concentrate on the local properties at the centre of the supercell whose size is rather large. There is no straight-forward rule for constructing a super-cell and in realistic calculations it becomes computationally expensive. A large number of generalizations of the CPA are beset with analytical difficulties and their effective medium is often not translationally symmetric. The only really successful generalization with analytical and translational properties is the traveling cluster approximation of (Kaplan et al., 1981).

\footnotetext{
* Corresponding Author: E-mail: salam7862007@yahoo.com
} 
Augmented space theorem and the recursion method [6] carried out in a minimal basis set of the tight-binding linear muffin-tin orbitals method (TB-LMTO) (Andersen et al., 1994; Andersen et al., 1984 and Andersen 1971) in which the Hamiltonian is sparse. The AST states that the configuration average of a well behaved function of a set of random variables is a particular matrix element of the operator obtained by replacing the random variables in the function by the corresponding operators, whose spectral densities are the probability densities of the random variables. The under-lying space in which the operator is defined is the space of all possible configurations of the random variables. For example, if the set of random variables have binary distributions, then this configuration space is isomorphic to the configuration space of a set of Ising spin-half objects. The theorem is exact and approximations are introduced only in the calculation of matrix element. The recursion method with a terminator approximation allows us to take into account effects of random environments of a site. The size of this environment depends upon the number of recursion steps we can carry out exactly and the far environment is approximated by the terminator.

\section{Method}

The LMTO method for self-consistent calculations of electronic structures of solids was introduced by Andersen and Jepson (Andersen et al., 1984). It has been described in great detail in a recent monograph (Andersen, 1995]. We shall indicate here only those points which are of specific importance for the present work. We stress here that the transformation of the canonical LMTO into a first principles tightbinding method with a sparse Hamiltonian representation is essential for an effective use of the recursion technique which is the basis of our methodology.

In LMTO representation within atomic sphere approximation, the basis functions have the form

$$
\chi_{R L}^{\alpha}\left(r_{R}\right)=\phi_{R L}\left(r_{R}\right)+\sum \phi_{R^{\prime} L^{\prime}}^{\alpha \alpha}(r R) h_{R L, R^{\prime} L^{\prime}}^{\alpha}
$$

Where $\phi$ is the product of spherical harmonic and the solution $\phi_{v R L}\left(\left|r_{R}\right|\right)$ of the radial wave equation inside the sphere centered at R for a certain energy $E_{v R L}$, which is in principle arbitrary, but in the energy range of interest. The functions $\phi^{\natural}$ are linear combinations of the products $\phi$ and their energy derivatives $\phi^{\ltimes}$. The actual choice of how this linear combination is made determines the basis. The matrix $h$ is given by

$$
h^{\alpha}=C^{\alpha}-E_{v}+\left(\Delta^{\alpha}\right)^{1 / 2} S^{\alpha}\left(\Delta^{\alpha}\right)^{1 / 2}
$$

Where $C^{\alpha}$ and $\Delta^{\alpha}$ are the diagonal potential parameter matrices. They depend on the potentials inside the atomic spheres, the representation $(\alpha)$ chosen and on the atomic sphere radii. The S matrix is a structure matrix which depends only on the representation $(\alpha)$ and on the geometrical arrangement of the atomic sites. In term of canonical structure matrix $S^{0}, S^{\alpha}$ is given by

$$
S^{\alpha}=S^{0}\left(1-\alpha S^{\alpha}\right)^{-1}
$$

Where $\alpha$ denotes a diagonal matrix, specifying the representation.

In recursion calculations it is practical to work with an orthonormal sparse representation. For this purpose it is advantageous to work in the $\gamma$ representation. It must be noted, however, that the structure matrix in the $\gamma$ representation is itself random in an intrinsic way. It is useful therefore to rewrite the Hamiltonian in terms of that in the most localized (or $\beta$ ) representation. The Hamiltonian in the $\gamma$ representation, which is correct up to second order in $\left(E-E_{v}\right)$, is given by

$$
H^{2}=E_{v}+h^{\gamma}
$$

The overlap matrix in this representation is a unit, diagonal matrix and therefore it fulfills the orthogonality condition required for recursion purposes. Expansion of $h^{\gamma}$ inn terms of the most localized short-ranged $h_{\alpha}$ is given by

$$
h^{\gamma}=h^{\alpha}-h^{\alpha} o h^{\alpha}-\ldots
$$

Where the matrix $o$ is diagonal in $R L$ representation and its value is determined by the logarithmic derivative of the function $\phi$ at the sphere boundary. For a particular choice of $\alpha=\beta$ which are independent of crystal structure given by

$$
\beta=\left\{\begin{array}{ll}
0.3485 & l=0 \\
0.0530 & l=1 \\
0.0107 & l=2
\end{array}\right\}
$$

The screened structure constant $S^{\beta}$ becomes particularly short ranged with a universal exponential decay for different structures (fcc, bcc, hcp, etc). Due to the exponential behavior of the structure factor, even for s and p bands it is not necessary to consider interactions beyond second nearest neighbors. 
If the power series given by (2) is truncated after the firstorder term, we obtain a two-centre sparse Hamiltonian. This Hamiltonian is correct up to first order in $\left(E-E_{v}\right)$, and it has been shown that it gives satisfactory band structure description for most solids except those with very broad bands. (Andersen,1985). However, since each term in the second and subsequent terms in the expansion is themselves twocentered and sparse, their inclusion in the recursion method introduces no difficulty. In subsequent sections we will use the first-order, tight-binding two-centre form of the LMTO Hamiltonian given by

$$
H^{1}=C^{\beta}+\left(\Delta^{\beta}\right)^{1 / 2} S^{\beta}\left(\Delta^{\beta}\right)^{1 / 2}
$$

Again, both the augmented space formalism and recursion method have been described in great detail in earlier work (Andersen,1995; Maan et al.,1982). We shall only emphasize those points here which are of relevance to the present work and refer the reader to the articles referenced above for the details.

In the augmented space formalism, we construct a non-random Hamiltonian defined on a new enlarged Hilbert space, which is a direct product of the Hilbert space spanned by the original Hamiltonian basis set and the configuration space which is spanned by the various allowed configuration states of the disordered Hamiltonian. The augmented space theorem (Mookerjee,1973) then relates the configuration averaging to projection onto a particular subspace: the so-called sum space (Gray et al., 1976; Mayou et al., 1993 and Julien and Mayon et al.,1993). This configuration averaging in the augmented space is exact and does not involve any singlesite approximation as in CPA and treats both diagonal and off-diagonal disorder on an equal footing.

The whole process can be summarized in the following basic steps.

(a) Since the probability density $p_{i}\left(n_{i}\right)$ of random variable $n_{i}$ associated with the Hamiltonian is a positive semi-definite function and if we assume all its moments to be finite, we may find a self-adjoint operator $M^{(i)}$ in a configuration space $\phi^{(i)}$ such that $p_{i}\left(n_{i}\right)$ can be expressed as its spectral density.

$$
p_{i}\left(n_{i}\right)=-1 / \pi \operatorname{lm}\left(\left\langle v_{0}^{i}\left|\left(n_{i}-M^{(1)}\right)^{-1}\right| v_{0}^{i}\right\rangle\right)
$$

This is the inverse of the well known problem of obtaining a local density of states starting from a self-adjoint Hamiltonian H. If $p_{i}\left(n_{i}\right)$ can be expressed in continued fraction expansion, then the representation of $M^{(i)}$ is tri-diagonal matrix with continued fraction coefficients in diagonal and off-diagonal positions.

(2) The averaged quantity $\int f\left(n_{i}\right) p_{i}\left(n_{i}\right)$ can be shown to be given by the matrix element $\left\langle v_{0}^{i}\left|\tilde{f}\left(M^{(i)}\right)\right| v_{0}^{i}\right\rangle$ where $\tilde{f}$ is the same functional of $M^{(i)}$ as $f$ was of $n_{i}$.

(3) For more than one random variable we define a product of space $\Phi=\Pi^{\otimes} \phi^{(i)}$. This is spanned by states in which the set of variables $n_{i}$ assumes one of its configurations. The averaged quantity $\langle f\rangle$ is now given by $\left\langle v_{0}\left|\tilde{f}\left(M^{(i)}\right)\right| v_{0}\right\rangle$ where $\left|v_{0}\right\rangle=\Pi^{\otimes}\left|v_{0}\right\rangle^{i}$ spans the so-called sum space, which is subspace of $\Phi$. A little algebra will show us that $\left|v_{0}\right\rangle=\Pi_{i}^{\otimes}\left(\sum_{\lambda i} \sqrt{p_{\lambda i}}\left|\lambda_{i}\right\rangle\right) \quad$ where $\left|\lambda_{i}\right\rangle$ the eigenstates of $\tilde{M}^{(i)}$ and $\left\{p_{\lambda i}\right\}$ are the associated probability weights.

The calculation of $\langle f\rangle$ thus reduces to calculating a particular matrix element in the augmented space. For electronic structure calculations in a disordered system, $f$ is chosen to the green function $\left(z I-H\left(\left\{n_{i}\right\}\right)\right)^{-1}$ where $\mathrm{H}$ is the Hamiltonian of the system and $n_{i}$ are the random site occupation variables.

An efficient algorithm for calculating diagonal matrix elements of the resolvent or the green function is provided by the recursion method introduced by Haydock and coworkers et al., 1972. Given the starting vector $\left|\Psi_{0}\right\rangle=\left|i, v_{0}\right\rangle$ in augmented space one generates a discrete chain of vectors $\left|\Psi_{i}\right\rangle$ through the following set of equations.

$$
\begin{aligned}
& \tilde{H}\left|\Psi_{i}\right\rangle=a_{i}\left|\Psi_{i}\right\rangle+b_{i+1}\left|\Psi_{i+1}\right\rangle+b_{i}\left|\Psi_{i-1}\right\rangle \\
& b_{0}^{2}=\left\langle\Psi_{0} \mid \Psi_{0}\right\rangle \\
& b_{i}^{2}=\left\langle\Psi_{i}|\tilde{H}| \Psi_{i+1}\right\rangle \\
& a_{i}=\left\langle\Psi_{i}|\tilde{H}| \Psi_{i}\right\rangle
\end{aligned}
$$

Where $\tilde{H}$ is the operator defined in the augmented space which is constructed by substituting the random site occupation variables $\left\{n_{i}\right\}$ by their associated self-adjoint operator $\left\{M^{(i)}\right\}$. 
This prescription essentially transforms the effective Hamiltonian $H$ to a trigonal form and thus lead directly to a continued fraction representation for the averaged green function matrix element $\left\langle\Psi_{0}|G| \Psi_{0}\right\rangle=\left[G_{i i}\right]_{a \dot{v}}$ If the algorithm is stop after $L$ steps, $L$ exact levels of the continued fractioned are obtained. The recursion algorithm after $L$ steps contains a contribution only from the central cluster containing $O\left(L^{3}\right)$ sites in the augmented space. For eliminating such a finite-cluster effect $L$ steps of recursion coefficients are appended with a terminator which mimics the asymptotic part of the continued fraction.

\section{Computational Details}

The densities of states have been found by the recursion method with the augmented space Hamiltonian. The augmented space map is generated from a real space cluster of 400 atoms. We have generated a sequence of eight couples of continued fraction co-efficient for s, p and d states and the terminating scheme of Lucini and Nex (Lucini et al., 1994) has been used. Regarding the issue of charge self-consistency we have followed the approximate yet accurate and consistent scheme of charge self-consistency proposed by (Andersen et al.,1987). Here the charge neutrality is achieved by exploiting the flexibility in the choice of sizes of Wigner-Seitz spheres of constituents in binary alloys. It involves scaling of the atomic sphere radii of alloy species with the aid of volume derivative correction in such a way that the spheres are approximately charge neutral, a fact discussed in great detail by Kudrnovsky/ and Drchal (Kudrnovsky and Drchal, 1990).Though the augmented space formalism has been recognized as a powerful tool for configuration averaging, its implementation so far has been restricted to model systems. This was because standard recursion cannot deal with the large rank of the augmented space. In particular, the nearest-neighbor map which has to be initially stored for any recursion is too large for storage and manipulations on this map are time consuming. We have reduced this problem to a tractable one by an explicit use of bit manipulation techniques and reduction of the large augmented space to a manageable irreducible subspace on which the recursion could be carried out. As these points have already been described in detail elsewhere (Saha et al.,1995) we will only mention the salient points. The basis vectors defined in the configuration space, carrying information about the occupation variable at each site, are strings of zeros and ones. This allows us to extensively borrow the
Ising computational methodology (Chowdhury et al., 1987) which involves storage of configuration states leads to largescale saving of disk space while the use of logical operations to describe the action of the augmented space Hamiltonian. The binary word representation of configuration states leads to large scale saving of disk space while the use of logical operations makes the computation faster. The work-load of augmented space recursion is further reduced by exploiting the symmetry of the Hamiltonian. The symmetry of the Hamiltonian arises from that of the underlying lattice and from homogeneity of disorder. It has been shown by Gallagher that if the starting state of recursion belongs to the irreducible representation of the Hamiltonian then the states generated in the process of recursion belong to the same row of the same irreducible representation of the Hamiltonian, so one needs to retain only those states for the purposes of recursion and get the same resolution as with all of them. Since the augmented space recursion retains all the properties of the real space recursion, the confinement of the recursion procedure to the irreducible portion of the Hamiltonian holds good for augmented space recursion also. The basic step in the symmetry procedure is to identify the set of nonequivalent vectors and their weights which can be achieved in augmented space by applying point point group symmetry operations to real space and configuration space by applying point group symmetry operations to real space and configuration space independently. This reduces the rand of the augmented space Hamiltonian drastically, reducing the computer storage and, at the same time, increasing the computer speed.

\section{Results and Discussion}

We have applied our methodology, discussed in earlier section to calculating the densities of states of $\mathrm{Ni}$ alloys for $10 \%, 20 \%, 30 \%, 40 \%, 50 \%$ and $60 \%$ concentration of $\mathrm{Cu}$ and $80 \%$, 88\%, 90\%, 92\%, 93\%, 94\% 95\%, 96\%, 97\%, 98\%, $99 \%$ concentration of Au per mole. Densities of states of $\mathrm{NiCu}$ and NiAu alloys with up and down spin configuration have been put.

Figure 1 to 3 shows the density of states of NiCu alloys with up and down spin configurations. Here, two separated peaks due to $\mathrm{Ni}$ and very little deviation due to alloying with $\mathrm{Cu}$ are observed. A good deal of variations in the positions and width of the peak and some significant differences in the up and down spin configuration are observed. This trend indicates the on set of the magnetic phase of $\mathrm{Cu}$ alloys at some preferred concentrations. There is a broad peak in the density 

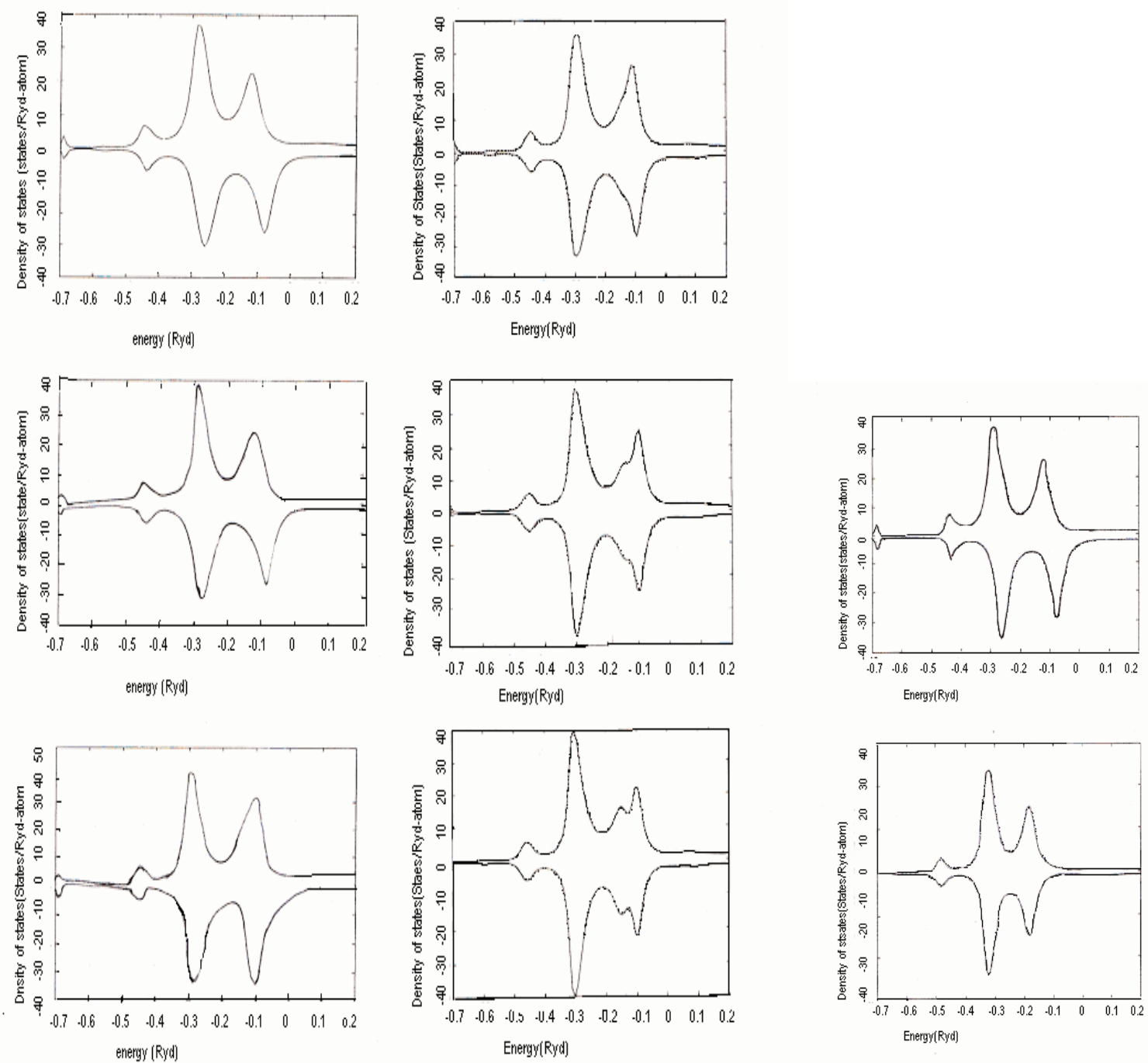

Fig.1. Density of states for $\mathrm{Cu}_{10} \mathrm{Ni}_{90}(\mathrm{top}), \mathrm{Cu}_{20} \mathrm{Ni}_{80}$ (mid dle) and $\mathrm{Cu}_{30} \mathrm{Ni}_{70}$ (bottom)

Fig. 2. Density of states for $\mathrm{Cu}_{40} \mathrm{Ni}_{60}$ (top), $\mathrm{Cu}_{50} \mathrm{Ni}_{50}$ (middle) and $\mathrm{Cu}_{60} \mathrm{~N}_{\mathrm{i} 40}$ (bottom)
Fig. 3. Density of states for NiNi (top) and $\mathrm{CuCu}$ (bottom) of states and no deviation due to change in concentration are observed. These figures show single prominent peak but with changes in the height and width of the peak due to change in the concentrations.

Figure 4 shows the magnetic moment of $\mathrm{NiCu}$ alloy as a function of concentration. At first the magnetic moment of $\mathrm{NiCu}$ alloy increases linearly and then decreases sharply. It is observed that the magnetic moment is increased with increasing the concentration of $\mathrm{Ni}$.

Figure 5 shows the plot of Fermi energy as function of magnetic constituents of $\mathrm{Ni}$ and $\mathrm{Cu}$. The exact behavior of Fermi energy depends sensitively on the exact shapes of the density of states and band filling. At first the Fermi energy decreases from a definite value of about -0.94 Rydberg with increasing the concentration of $\mathrm{Ni}$. After reaching to a lowest value of about -0.123 Rydberg, Fermi energy increases with increasing the concentration of $\mathrm{Ni}$. This happens because average number of electrons decreases with decreasing concentration of $\mathrm{Ni}$.

Figure 6 shows the plot of binding energy as function of concentrations of Ni. The binding energy of a electron in an alloy can be calculated from the following relation. 


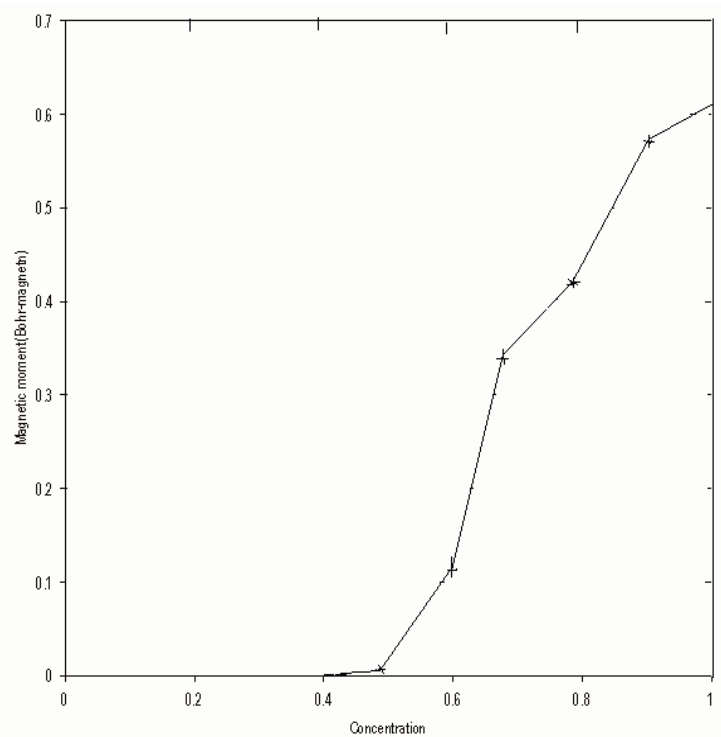

Fig. 4. Magnetic moment at different alloy concentration of CuNi

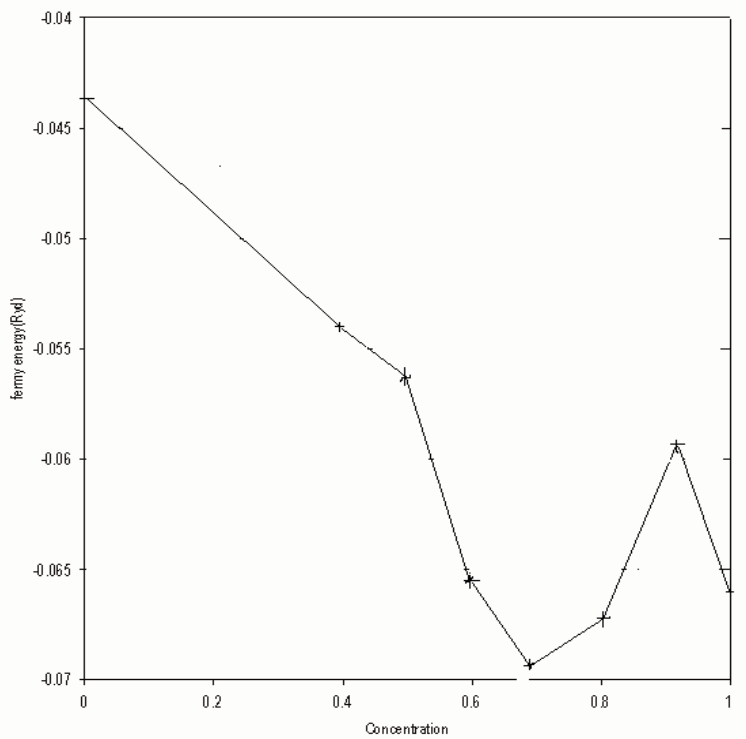

Fig. 5. Fermi energy at different alloy concentration of CuNi

$$
\Delta E_{\text {bind }}=E_{\text {total }}^{\text {alloy }}-\left\{x E_{A}^{a t o m}+(1-x) E_{B}^{a t o m}\right\}
$$

where, $\quad E_{A}^{\text {atom }}=\int_{-\infty}^{E_{F}} n(E) E d E$

$$
\text { And, } n(E) \approx n^{\uparrow}(E)+n^{\downarrow}(E)
$$

The binding energy of NiCu alloys increases monotonically with increasing the mole fraction of $\mathrm{Ni}$. It means that the

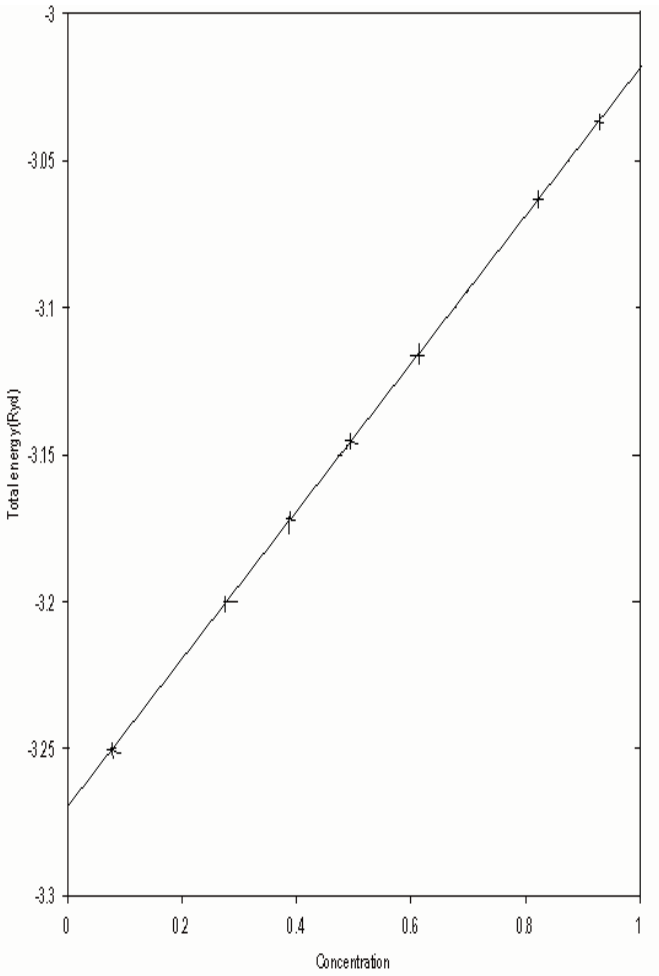

Fig. 6. Total energy at different alloy concentration of $\mathrm{CuNi}$

high concentration of $\mathrm{Ni}$ is more stable than the low concentration of $\mathrm{Ni}$ in $\mathrm{NiCu}$.

In figures 7 to 10 , distinct peaks of Ni-system with very little deviation due to alloying with Au are observed. A single prominent peak is observed. The shifting of peaks at different concentrations is observed for up and down spin cases. The first thing to note is that the s-d bands of $\mathrm{Ni}$ and $\mathrm{Au}$ do not overlap much.

Consequently in the low concentration regimes of either $\mathrm{Ni}$ or Au we have impurity like peaks of the dilute constituents are noticed. The densities of states are hardly spin split and straddle. As concentration of Au increases, the up spin band almost remains fixed, while the down spin band shifts upward in energy.

Figure 11 shows the magnetic moment of NiAu alloy as a function of Ni concentrations. It is observed that the magnetic moment is increased with increasing the concentration of $\mathrm{Au}$ and with decreasing the concentration of $\mathrm{Ni}$. It is notable that Au carries negligible magnetic moment. When the concentration of Ni increases, magnetic moment decreases linearly and ends at the point where magnetic moment of AuNi alloy are zero which is actual value. 

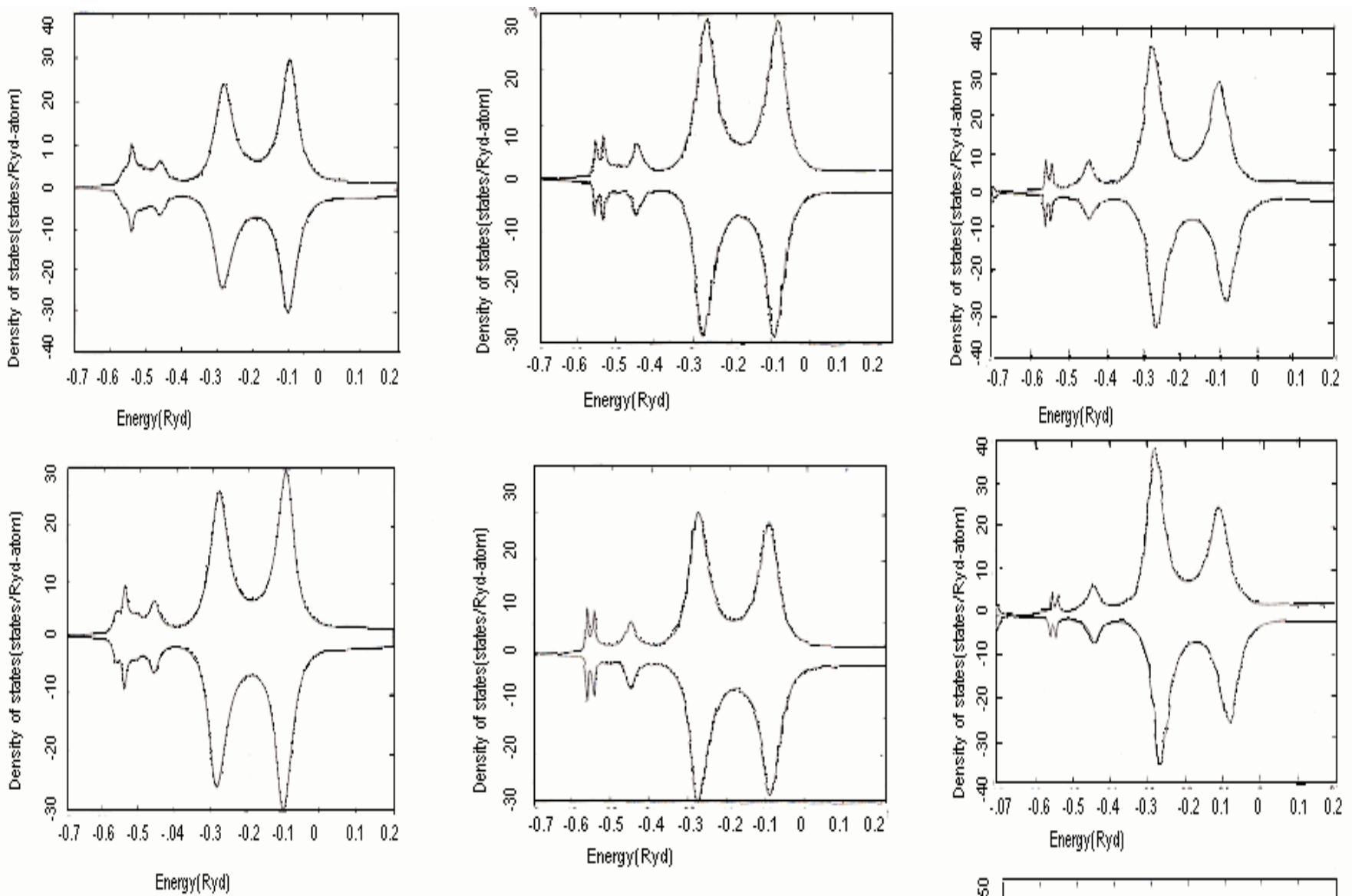

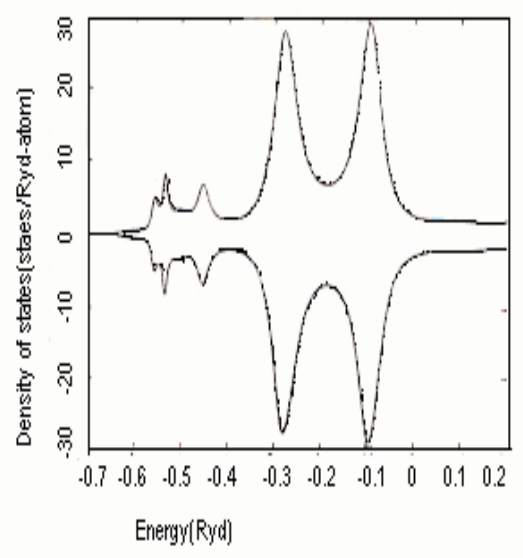

Fig .7. Density of statea of to NIAu(8812) Middle NiAu (9010) and bottom NIAu (9208)

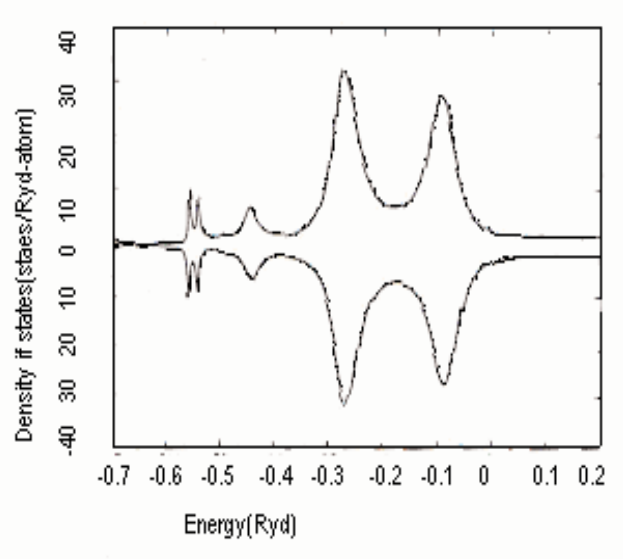

Fig. 8. Density of states for top NiAu (9307), middle NiAu(9406) and bottom NiAu (9505)

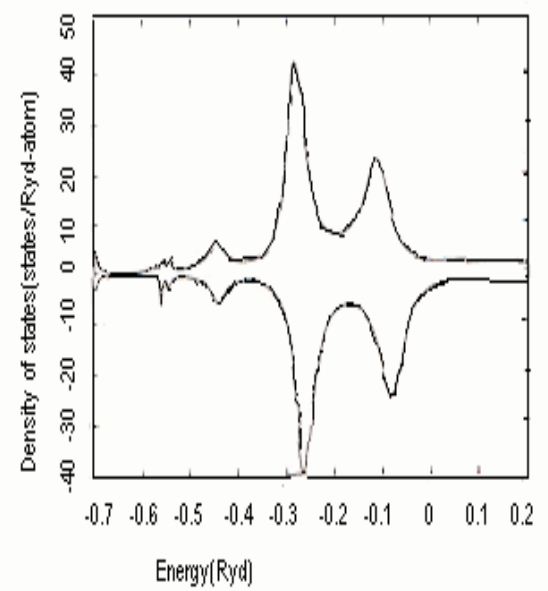

Fig. 9. Density of states for NiAu (9604) top NiAu(9703) middle, NiAu (9802)bottom 

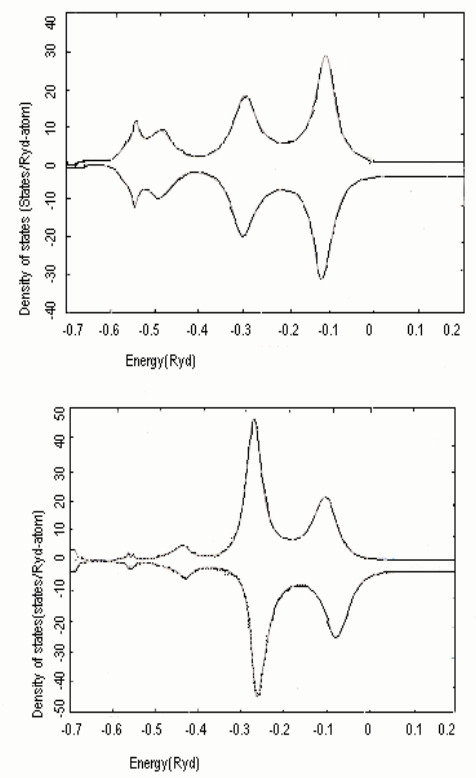

Fig. 10. Density of states for top NiAu(8020) and bottom NiAu(9901)

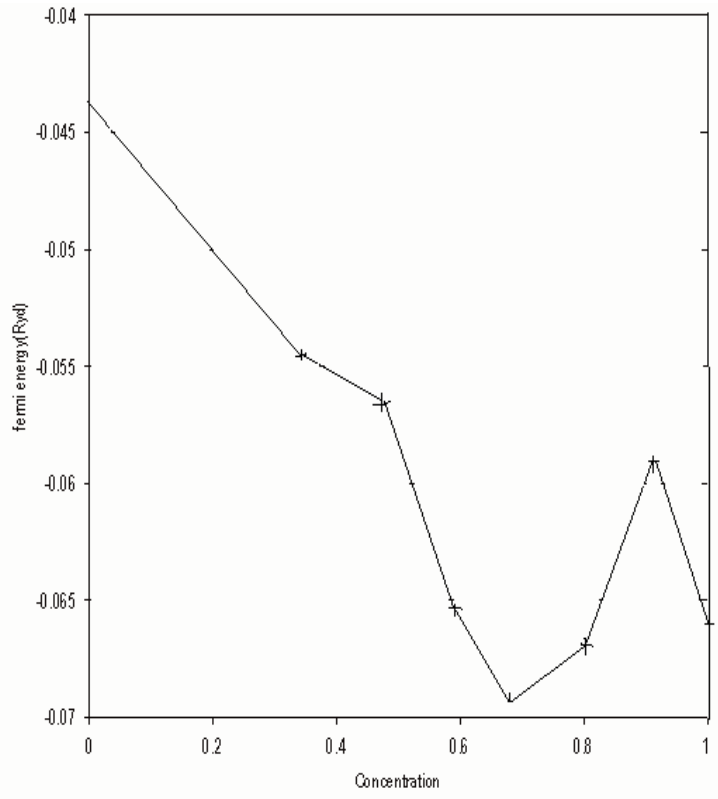

Fig. 12. Fermy energy at different alloy concentration of $\mathrm{NiAu}$

Figure 12 shows the plot of Fermi energy as a function of concentration of $\mathrm{Au}$ and $\mathrm{Ni}$. The exact behavior of the Fermi energy depends sensitively on the exact shapes of the density of states and band filling. Since Au bands lie higher in

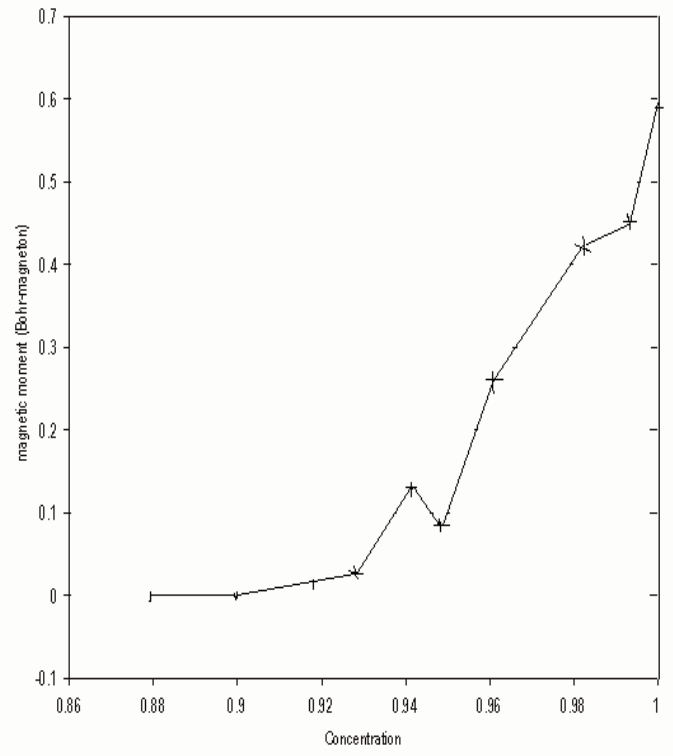

Fig. 11. Magnetic moment at different alloy concentration of AuNi

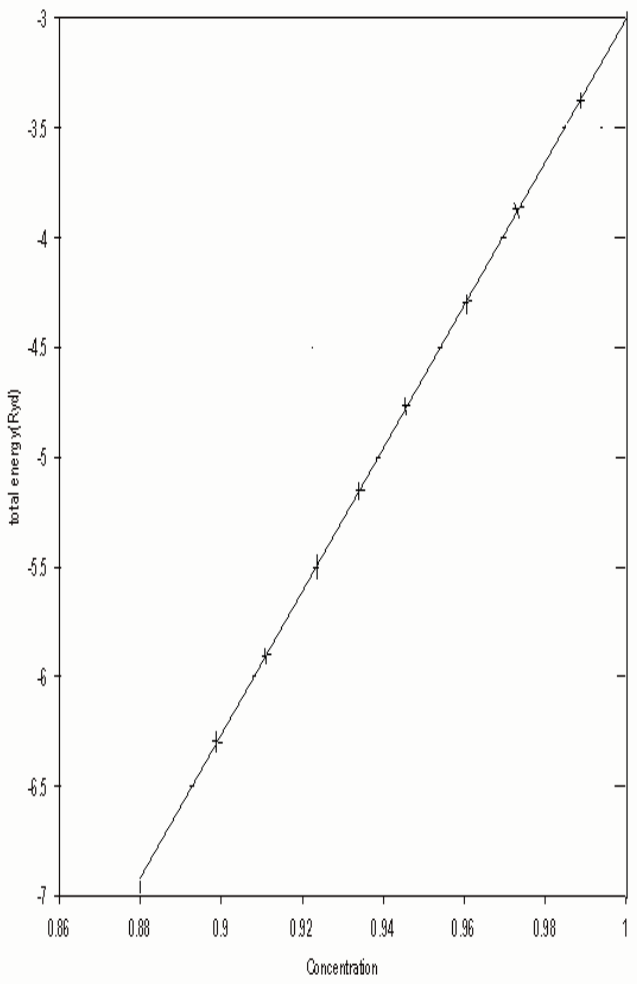

Fig. 13. Total energy at different alloy concentration of AuNi 
energy than $\mathrm{Ni}$, increasing Au concentration leads to upward shift in the Fermi energy.

Figure 13 shows the plot of binding energy as a function of concentrations of $\mathrm{Au}$ and Ni. Since Au bands lie higher in energy than $\mathrm{Ni}$, Increasing Au concentration lead also to upward shift in the binding energy. The binding energy of AuNi increases with increasing the concentration of Au. It means that the high concentration of Au is more stable than the low concentration of $\mathrm{Au}$ in AuNi.

A set of consistent data have been produced by the efficient, reliable and fast TB-LMTO method on the Ni-based alloys of $\mathrm{Au}$ and $\mathrm{Cu}$. The all have peaks in the density of states and these are consistent with the previous calculations (Bratkovsky et al., 1993).

\section{Conclusion}

The electronic structure of NiCu and AuNi alloys using fully self-consistent first principles electronic structure technique have been calculated and obtained results agrees reasonablywell with the experimental results (Bratkovsky et al., 1993). This clearly shows that ASR coupled with TB-LMTO is a powerful technique in describing electronic structure of binary alloys. Results clearly show the flaws in the theoretical results based upon model calculations. The band structure can be computed from here. These studies are going to be helpful for experimentalist and metallurgists, who would design materials and alloys with specific properties.

\section{References}

Abrikosov I. A., Niklasson A. M. N., Simal S. I., Johansson B., Ruban A. V. and Skriver H. L. (1996) Order- N Green's Function Technique for Local Environment Effects in Alloys' , Phys.Rev. Lett 76: 4203 -4210.

Anderson O. K., Jepsen O. and Krier G. (1994) Lecture on Methods of Electronic Structure Calculation ,edited by V. Kumar, O. K Andersen, A.Mookerjee, (world Scientific, Singapore), pp-63 .

Anderson O. K. and Jepsen O (1984) Explicit, FirstPrinciples Tight-binding Theory, Phy. Rev. lett. 53: 2571-2576.
Andersen O. K. (1971) Computational Methods in Band theory, edited by P. M. Marcus, J. F. Janak, A. R. Wiliams (Plenum, New York, 1971), pp.178.

Anderson O. K. (1995) Electronic Structure of Metals and Alloys, edited by V. Kumar , O.K Andersen, A. Mookerjee, (World Scientific, Singapore, 1995), pp199.

Anderson O. K., Jepsen O. $S^{\sim}$ and Glotzel D. (1985) Highlights of Condensed Matter Theory', edited by F. Bassani, F. Fumi and M. P. Tosi (New York: NorthHolland, 1985).

Andersen O. K., Jepsen O. and ob M. (1987) Electronic Structure and its Applications' Vol. 283, edited by M. Yussoff (Berlin: Springer), pp-413.

Bratkovsky A. M. and Smirnov A. V. (1993) Real-spaceLMTO spin-polarized self-consistent calculations of the electronic structure of the amorphous alloys $\mathrm{Ni}-\mathrm{B}$, Fe-B and Zr-Be', J. Phys: Condensed Matter 5: 32033220.

Chowdhury D., Gawlinski E. D. and Gunton J .D. (1987) Interface roughening and domain growth in the dilute Ising model', Phys. Rev. B 35: 6792 - 6795.

Gray J. L. and Kaplan T. ( 1976) Elementary excitations in random substitutional alloys', Phys. Rev. B 14 : 646270 .

Haydock R., Heine V. and Kelly M. J. (1972) Electronic structure based on the local atomic environment for tight-binding bands' J. Phys. C: Solid state Phys. 5: 2845-2850.

Julien J. P. and Mayou D. (1993) Solution of self-consistent field equations by the recursion method, J. Physique I 3: 1861-1869.

Kaplan T., Leath P. L., Gray L. J. and Diehl H. W. (1981) Self-consistent theory for random alloys with shortrange order', Phys. Rev. B 24: 1872-1880.

Kudrnovsky J. and Drchal V. (1990) 'Electronic structure of random alloys by the linear band-structure methods', Phys. Rev, B 41: 7515-7525. 
Lucini M. U. and Nex C. M. M (1994) Augmented-space recursive method for the study of short-ranged ordering effects in binary alloys, Phys. Rev. B 50:13267 - 13275.

Mann A. and Privman V. (1982) Localized SymmetryAdapted Perturbation Theory and a New Tight-Binding Expansion' Phys. Rev. Lett. 49:1068 - 1071.

Mookerjee A. (1973) Averaged density of states in disordered systems' J. Phys. C: Solid State Phys 6:1340.

Mayou D., Pasturel A. and Ngyuen Manh D. (1993)' The effects of local lattice distortion in non-isochoric alloys ...CuPd and CuBe'. J. Physique C 19: 719-723.
Pinski F. J., Ginatempo B., Johnson D. D., Staunton J. B., Stocks G. N. and Gyorffy B. I. (1991) 'Origins of compositional order in NiPt alloys', Phys.Rev. lett. 66: 766 - 775.

Saha T., Dasgupta I. and Mookerjee I. (1995) 'Augmentedspace recursive technique for the analysis of alloy phase stability in random binary alloys' Phy Rev B 51: 3413-3420.

Received : August 13, 2008;

Accepted : February 11, 2009 